\title{
Subcycle dynamics in the laser ionization of molecules
}

October 2007

\author{
Xinhua Xie, ${ }_{1}^{1}$ M. Wickenhauser, ${ }^{1}$ W. Boutu, ${ }^{2}$ H. Merdji, ${ }^{2,3}$ P. Salières, ${ }^{2}$ and A. Scrinzi ${ }^{1, *}$ \\ ${ }^{1}$ Photonics Institute, Vienna University of Technology, Vienna, Austria \\ ${ }^{2}$ CE Saclay, CEA, 91191 Gif Sur Yvette, France \\ ${ }^{3}$ Stanford PULSE Center, SLAC, Menlo Park, California 94025, USA
}

\begin{abstract}
The time and momentum distributions of electron emission from a molecule during a single laser cycle are calculated by solving a two-dimensional time-dependent Schrödinger equation. The momentum distributions strongly depend on the orbital symmetry and orientation of the molecular axis. Field-induced internal dynamics of the molecule can shift electron emission and recollision times through a large part of the laser cycle, which leads to corresponding variations of high-harmonic emission times and to the appearance of even harmonics.
\end{abstract}

\section{INTRODUCTION}

During a single optical cycle of a strong laser pulse, electrons are driven out of a molecule, accelerated, and directed back onto their parent molecule, where they scatter or recombine. This "recollision" process was first introduced to explain the generation of high-harmonic radiation $[1,2]$, but it also leads to the appearance of electron diffraction patterns $[3,4]$, or the expulsion of more electrons and disintegration of the molecule [5]. As electron detachment and recollision occupy only a fraction of the whole cycle, time structures are on the scale of a few hundred attoseconds. These extremely rapid processes can be manipulated by controlling the laser field, and this is being exploited in an increasing number of experiments. From high-harmonic radiation attosecond pulses can be extracted, and efforts are directed to obtaining higher-harmonic intensities and detailed control of the time structure. Harmonic radiation also serves to diagnose recollision itself and to extract information about the structure [6] and dynamics of the target $[7,8]$. The early focus of recollision experiments was on ionization and harmonic generation with noble gases, where the recollision picture has become firmly established. The chirp of the harmonics can be linked to recollision times [9], and control of the recollision through laser polarization $[10,11]$ and two-color fields [12] have been demonstrated. Now attention is increasingly shifting toward molecules, because of their more complex structure and richer dynamics. The orientation of the molecule relative to the laser field is an additional experimental parameter with which, e.g., the ionization yield can be varied significantly. The basic features of the orientation dependence of total ionization are predicted by the molecular Ammosov-DeloneKrainov (ADK) theory of tunnel ionization [13], which depends only on the asymptotics of the field-free electronic wave function in the field direction.

To determine the momentum distribution of the emitted electrons, knowledge of the wave function in a fixed direction is insufficient: in the simplest case of a diatomic molecule, electrons are released from around both centers, leading to three-dimensional interference patterns that depend on

\footnotetext{
*scrinzi@tuwien.ac.at
}

the relative phases and positions of the two centers [14]. More generally, the emission depends on the nodal structure of the outer electronic orbitals [15]. For the time of electron release, electronic dynamics during the emission process must be taken into consideration. In its usual form, the recollision picture assumes that ionization is a quasistationary process, where at any time the rate is proportional to the tunnel ionization rate for the instantaneous field strength. Because of the strongly nonlinear dependence of ionization on field strength, this implies a sharp maximum of emission at the peak of the laser field. However, ionization can be quasistationary only when the time scales of electronic motion in the system are well separated from the laser time scale. In molecules, this is not generally the case.

In this paper, we investigate the influence of molecular electronic structure and dynamics on electron emission and recollision. We introduce a pseudodetector method to define emission and recollision times as well as electron momenta after emission within the frame of the time-dependent Schrödinger equation (TDSE). For two-dimensional model molecules, we find pronounced effects of orientation on both electron spectra and emission times, due to the orbital symmetry and field-induced intramolecular dynamics.

\section{METHOD}

To define a time of ionization and an electron spectrum at that time, one needs to distinguish between bound and unbound electrons while the field is present. This is discussed in detail in Ref. [16], where an approximate bound-unbound distinction was introduced. This distinction is applicable in the case of strong fields, while at weaker fields it becomes dependent on details of the approximation, in particular on the gauge used to describe the interaction with the field. For the present work, we avoid this problem by adopting the original idea of the classical recollision model for the timedependent Schrödinger equation. The electron detachment is determined from the wave function amplitude that we find at distances where the laser force dominates over the molecular potential. We can associate that amplitude with a time of electron emission by propagating it back to near the ion. In this way, we can discuss electron recollision without the need 


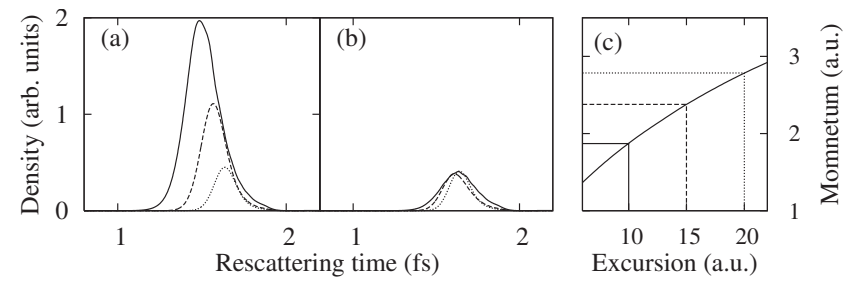

FIG. 1. Time distribution of density of recolliding electrons on target as obtained with probe distances $z_{0}=10$ (solid line), 15 (dashed line), and 20 a.u. (dotted line). In (a) all recollision momenta are included; in (b) momenta are chosen such that all electrons reach 20 a.u. (c) shows the relation between recollision momentum and excursion amplitude for short trajectories.

to decide whether the electronic wave function ever became unbound or just strongly distorted.

Here we summarize the implementation of the idea and we refer the reader to Ref. [17] for details. At time $t_{0}$ the solution $\Psi(\vec{r}, t)$ of the TDSE is multiplied by a probe function $M_{z_{0}}(\vec{r})$ which is localized at a distance $z_{0}$ from the bound system measured in the polarization direction. This probe function approximates a plane where we measure the electron density and momentum as a function of time. $M_{z_{0}}$ is chosen as a Gaussian function centered around $z_{0}$ in the $z$ direction with widths between 3 and 7 a.u. The extension in the perpendicular direction was chosen much wider with a typical value of 20 a.u. The probed part of the wave function $\chi_{t_{0}}\left(t_{0}\right)=M_{z_{0}}(\vec{r}) \Psi\left(\vec{r}, t_{0}\right)$ is propagated further by the TDSE,

$$
i \frac{\partial}{\partial t} \chi_{t_{0}}(t)=H \chi_{t_{0}}(t)
$$

By integrating over all probe times $\Phi_{r}(\vec{r}, t)=\int d t_{0} \chi_{t_{0}}(\vec{r}, t)$, one obtains the recolliding part of the wave function, from which the density distributions in time, space, and momentum "on target," i.e., at $z=0$, are calculated. In Ref. [17] we show that the currents on target only weakly depend on the probe distance $z_{0}$ and the width and shape of the mask function. This allows one to identify the times of electron release and recollision. Here one must take into account that with increasing probe distance fewer electrons reach the probe. For emission times corresponding to the "short trajectories" of the classical recollision picture [18] there is a one-to-one correspondence between the classical excursion amplitude and the recollision momentum. By filtering only the highest recollision momenta, one can make sure that all recolliding electrons have passed through the probe. Results for probe distances $z_{0}=10,15$, and 20 a.u. are shown in Fig. 1. The calculations were performed for the two-dimensional molecular model described below with a symmetric initial state, laser polarization perpendicular to the molecular axis, $800 \mathrm{~nm}$ wavelength, and a peak laser field strength corresponding to intensity $1.4 \times 10^{15} \mathrm{~W} / \mathrm{cm}^{2}$. When all probed electrons are included, recollision densities are strongly enhanced at early return times for the shorter probe distances. However, these are low-momentum electrons. When one counts high-momentum electrons that pass through all respective barriers, the time distributions nearly agree [Fig. 1(b)]. Low-energy trajectories that miss all probe functions belong to a regime where the concept of recollision ceases to be applicable. Dependencies on the probe width and on the gauge in the TDSE were found to be similarly weak [17].

\section{RESULTS AND DISCUSSION}

In the following, we study electron emission and recollision using a two-dimensional single-electron model with screened Coulomb potentials

$$
H=-\frac{1}{2}\left[\vec{\nabla}_{\rho}-\vec{A}(t)\right]^{2}-\sum_{s= \pm 1 / 2} \frac{c}{\sqrt{a^{2}+(\vec{\rho}+s \vec{R})^{2}}},
$$

with the coordinates $\vec{\rho}=(x, z)$ and the molecular axis at an angle $\theta: \vec{R}=(R \sin \theta, R \cos \theta)$ and internuclear separation $|R|=4.4$ a.u. For the potential parameters we chose $c=0.5$, and $a$ was adjusted to obtain an ionization potential of 0.51 a.u. This choice of $c$ implies a symmetric molecule with Coulombic long-range behavior of the potential, while $|R|$ and the ionization potential mimic the overall size and ionization potential of $\mathrm{CO}_{2}$. The vector potential was chosen in the $z$ direction $\vec{A}=\left(0,\left(E_{0} / \omega_{0}\right) \cos \omega_{0} t\right)$ with peak field strength $E_{0}$ and center frequency $\omega_{0}=0.057$ a.u. $(\lambda=800 \mathrm{~nm})$. For our calculations we can neglect nuclear motion, as electron release and recollision happen during about a laser half cycle of $1.3 \mathrm{fs}$, which should be compared to typical vibrational periods of $15-25$ fs for molecules like $\mathrm{CO}_{2}$. Although the two-dimensional model precludes quantitative comparisons with experimental data, we will argue below that the mechanism underlying the release dynamics is universal and not specific for our model. The TDSE with the Hamiltonian (2) was solved by a pseudospectral method with a box size of 40 and 120 a.u. in the $x$ and $z$ directions, respectively. The ground and excited initial states were obtained by imaginary time propagation, where for the excited state orthogonality with respect to the previously calculated ground state was imposed. Starting from the ground state, we determine electron momenta through a barrier at $z_{0}=15$ a.u.

\section{A. Electron momentum distributions}

The upper panels of Fig. 2 show the electron momentum distribution for orientations $\theta=0^{\circ}, 45^{\circ}$, and $90^{\circ}$ in directions parallel $(g, \|)$ and perpendicular $(g, \perp)$ to the laser polarization. The distributions are given for the times, when the electron density on the probe reaches its maximum. In qualitative agreement with the molecular ADK theory [13], the ionization yields decrease with increasing angle. The overall shapes of the parallel and perpendicular momentum distributions remain similar and smooth. For parallel alignment, the distribution is shifted to slightly higher momenta, which can be related to a variation in the electron emission time (see the discussion below). In the perpendicular momentum distribution a slight asymmetry appears at $45^{\circ}$. This picture changes drastically when we ionize from a state with ungerade symmetry. We use the first excited state of a system, where for easier comparison with the gerade state we adjust the potential parameter $a$ in the Hamiltonian (2) to obtain the same 


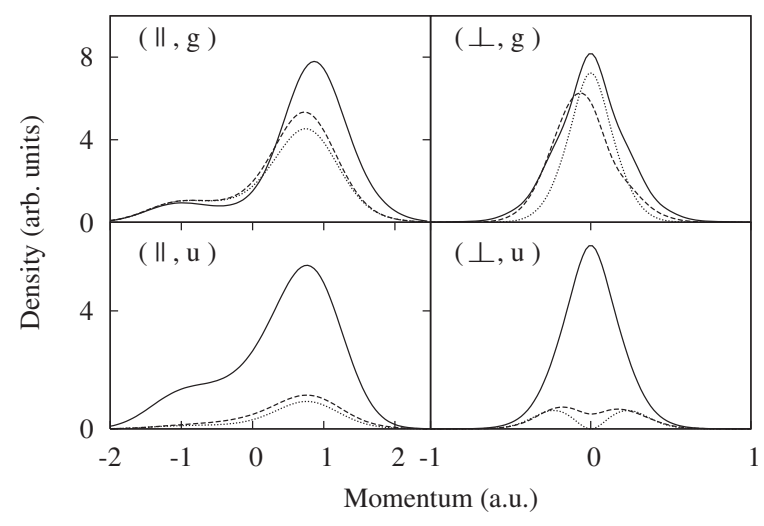

FIG. 2. Dependence of the electron momentum distributions at $z_{0}=15$ a.u. on molecular orientation and initial state symmetry. Upper panels: parallel $(\|, g)$ and perpendicular $(\perp, g)$ momentum distributions with initial gerade states for parallel $\theta=0^{\circ}$ (solid line), $\theta=45^{\circ}$ (dashed line), and perpendicular $\theta=90^{\circ}$ (dotted line) alignment of the molecular axis. Lower panels: same for an antisymmetric initial state.

binding energy of 0.51 a.u. The lower panels of Fig. 2 show the probed spectra for the excited state with ungerade symmetry. In this symmetry, the ionization yield drops more strongly when going from parallel to perpendicular orientation. More importantly, the distribution of perpendicular momenta changes its shape from a smooth bell-shaped distribution to a double-hump distribution with a node for perpendicular momentum $p_{\perp}=0$. The appearance of this node follows immediately from the antisymmetry perpendicular to the polarization direction.

The suppression of zero perpendicular momentum leads to strong suppression of recollision and high-harmonic generation in addition to the suppression of total ionization. The effect must also be taken into account for a correct interpretation of the experiments $[5,19]$, where double ionization by recolliding electrons was used as a measure of orientationdependent ionization rates. Some of the molecules investigated there have exact antisymmetries and all have nodal planes which will lead to strong variations of the momentum distributions of the recolliding electrons with molecular orientation. The observed double ionization therefore depends not only on total ionization, but also on the fraction of the returning electrons that contribute to double ionization. For example, while for our model and parallel orientation $\sim 50 \%$ of the returning electrons pass through a region around the molecule of twice the internuclear distance, this fraction is only $\sim 10 \%$ for the perpendicular orientation.

\section{B. Electron emission and recollision times}

The time structure of electron emission is even more deeply connected to molecular orientation and structure. Figure 3 (left panels) shows the density of emitted and recolliding electrons for a range of intensities and parallel alignment of the molecular axis with the laser polarization. The emission time structure varies strongly with intensity. At intensity $5.6 \times 10^{13} \mathrm{~W} / \mathrm{cm}^{2}$, peak emission approximately coincides with peak field strength, but at intensity $1.3 \times 10^{14} \mathrm{~W} / \mathrm{cm}^{2}$
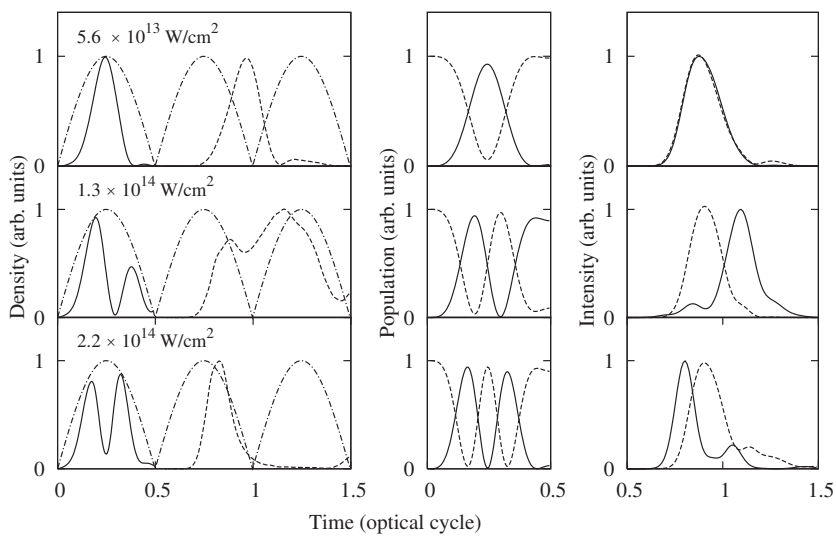

FIG. 3. Time distributions of electron emission and recollision (left panels), state populations (center), and harmonic emission (right) for laser intensities $5.6 \times 10^{13}, 1.3 \times 10^{14}$, and $2.2 \times 10^{14} \mathrm{~W} / \mathrm{cm}^{2}$ (from top to bottom). The dot-dashed lines indicate $|\mathcal{E}(t)|$. Left panels: emission (solid) and recollision (dashed) distributions scaled to maximal values of 1 with the probe located at $z_{0}=6$ a.u., molecular alignment parallel to the laser field. Center panels: populations of ground state (dashed) and first excited state (solid), parallel alignment. Right panels: combined intensity of harmonics above $90 \%$ of the harmonic cutoff energy with parallel (solid) and perpendicular (dashed) alignment.

two emission peaks of different heights appear that are separated by approximately $1 / 4$ of an optical cycle, and at the largest intensity of $2.2 \times 10^{14} \mathrm{~W} / \mathrm{cm}^{2}$ two roughly equal peaks with slightly smaller separation appear.

Analyzing the electronic wave function during emission, one finds that the variations are caused by intramolecular electron dynamics induced by the ionizing field. Figure 3 (center panels) shows the populations of the (field-free) ground and first excited states during the ionizing laser half cycle. The laser field induces pronounced Rabi-like oscillations of the populations of the ground and the first excited state. Electron emission predominantly happens when the excited state becomes populated at times when the field is strong. In that way, emission itself becomes modulated by the oscillations between the bound states. The variations in the recollision times match emission, if one takes into account that only emission times corresponding to classically recolliding trajectories can contribute significantly. At the intensity $1.2 \times 10^{14} \mathrm{~W} / \mathrm{cm}^{2}$, the variation of emission times leads to a strong emphasis of "long trajectories," i.e., recollisions well after the node of the field. Note that emission can also peak before the peak of the laser field, when the excited state population becomes maximal before the peak field. We also investigated the emission time structure for laser polarization perpendicular to the molecular axis, where the excited state is not dipole reachable. As expected, the emission time structure closely follows the field strength and very weakly varies with intensity. Correspondingly, recollision occurs according to the classical model.

The mechanism makes it clear that the modulation of electron emission by field-induced internal dynamics is a universal phenomenon, which is not bound to the specific model used here. In general, the dynamics modifies ionization times when (1) it occurs on a time scale that is compa- 
rable to the laser optical period and (2) the internal rates are significant compared to the ionization rate. Condition (1) means that there is an electronically excited state at most a few photon energies above the ground state. At a laser photon energy of $1.5 \mathrm{eV}$ corresponding to the $\sim 800 \mathrm{~nm}$ wavelength, this condition becomes satisfied at large internuclear distances. Condition (2) can always be met by reducing laser intensity. At fixed wavelength, that means that one approaches Keldysh parameters of $\gamma \sim 1$, which is, in fact, where many experiments are being performed. Oscillations between the populations of bound states were reported also at very strong field [20], where, however, the oscillation frequency far exceeds the laser frequency and no observable modulation of the emission results.

\section{Experimental signatures}

The most sensitive experimental signature of recollision is in high-harmonic emission, where recollision momenta are closely linked to recollision times $[8,9]$. The experimental signature of field-induced intramolecular dynamics is the dependence on the laser intensity and orientation of the molecular axis. The right-hand panels in Fig. 3 show that the single-atom harmonic response in the energy range above $90 \%$ of the harmonic cutoff energy closely follows the corresponding recollision density distribution. The figures also contain the harmonic intensities for perpendicular orientation, which remain independent of intensity. Recent experimental work has revealed a similar change of the harmonic emission profile as a function of intensity and orientation of the molecular axis [21].
Another observable effect of the internal dynamics is the transient appearance of even harmonics. Between subsequent recollisions, the system evolves according to the fieldinduced dynamics. In our case, Rabi-like oscillations between the gerade ground and the ungerade excited state lead to variations of the electron density. At intensities where the ratio of the laser period to the field-induced internal oscillation period approaches an even number, the system returns to the same state at each subsequent recollision. Any asymmetry of this state will cause even harmonics. As the internal dynamics depends on laser intensity, the even harmonics show a resonancelike dependence on pulse intensity. For our model system, parallel alignment, a pulse duration of $15 \mathrm{fs}$, and intensity of $5.6 \times 10^{13} \mathrm{~W} / \mathrm{cm}^{2}$, we find even harmonic peaks with up to $3-8 \%$ of the peak height of the neighboring odd harmonic peaks. For perpendicular alignment there are no even harmonics. Finally, switching of recollisions between short and long trajectories (cf. Fig. 3) changes the phase-matching conditions. Depending on the position of the focus before or after the interaction region with the gas, complementary effects of the internal dynamics should be observed. A detailed investigation of these phenomena is deferred to future work.

\section{ACKNOWLEDGMENT}

This work was supported by the Austrian Research Fund project F016 and in part by the U.S. Department of Energy, through the Stanford PULSE Center at SLAC.
[1] P. B. Corkum, Phys. Rev. Lett. 71, 1994 (1993).

[2] K. C. Kulander, K. J. Schafer, and J. L. Krause, in Proceedings of the Workshop, Super Intense Laser Atom Physics (SILAP) III, edited by B. Piraux (Plenum, New York, 1993).

[3] H. Niikura et al., Nature (London) 417, 917 (2002).

[4] M. Lein, N. Hay, R. Velotta, J. P. Marangos, and P. L. Knight, Phys. Rev. A 66, 023805 (2002).

[5] A. S. Alnaser et al., Phys. Rev. Lett. 91, 163002 (2003).

[6] J. Itatani et al., Nature (London) 432, 867 (2004).

[7] H. Niikura et al., Nature (London) 421, 826 (2003).

[8] S. Baker et al., Science 312, 424 (2006).

[9] Y. Mairesse et al., Science 302, 1540 (2003).

[10] I. Mercer et al., Phys. Rev. Lett. 77, 1731 (1996).

[11] G. G. Paulus et al., Phys. Rev. Lett. 84, 3791 (2000).

[12] T. Remetter et al., Nat. Phys. 2, 323 (2006).

[13] X. M. Tong, Z. X. Zhao, and C. D. Lin, Phys. Rev. A 66,
033402 (2002)

[14] J. Muth-Böhm, A. Becker, and F. H. M. Faisal, Phys. Rev. Lett. 85, 2280 (2000).

[15] T. K. Kjeldsen, C. Z. Bisgaard, L. B. Madsen, and H. Stapelfeldt, Phys. Rev. A 71, 013418 (2005).

[16] O. Smirnova, M. Spanner, and M. Ivanov, J. Phys. B 39, S307 (2006).

[17] X. Xie, G. Jordan, M. Wickenhauser, and A. Scrinzi, J. Mod. Opt. 54, 999 (2007).

[18] P. Antoine, A. L'Huillier, and M. Lewenstein, Phys. Rev. Lett. 77, 1234 (1996).

[19] C. D. Lin, X. M. Tong, and Z. X. Zhao, J. Mod. Opt. 53, 21 (2006).

[20] T. Zuo and A. D. Bandrauk, Phys. Rev. A 54, 3254 (1996).

[21] W. Boutu et al. (unpublished). 